\title{
FREE LAYERS AND SINGULAR JUMPS IN SOME SINGULARLY PERTURBED BOUNDARY VALUE PROBLEMS WITH TURNING POINTS
}

\section{Adriana Bohé}

\section{Introduction}

Consider the singularly perturbed boundary value problem $P_{q}$ :

$$
\begin{gathered}
\varepsilon x^{\prime \prime}+g(x) x^{\prime q}=0, \quad 0<t<T, \quad\left({ }^{\prime}=\frac{d}{d t}\right), \\
x(0)=A, \quad x(T)=B,
\end{gathered}
$$

where $q=0$ or $1 \leq q \leq 2, \varepsilon$ is a fixed positive infinitesimal, $T, A$, and $B$ are standard values, $g(x)$ is a smooth standard function, and the interval $[A, B]$ contains zeros of $g(x)$.

Such boundary value problems arise in many different applied contexts as in the study of exit time problems for stochastic differential equations (cf. Matkowsky [11]) in the case $q=1$. Other applications of such problems occur when $q=0$, in reactiondiffusion and phase transition models (cf. Fife [8]), in the study of contrast structures (cf. Butuzov and Vasil'eva [4]), and also in problems related to critical paths of Feynman integrals (cf. McLaughlin [12, 13]).

The behavior of the solutions of (1), (2) presents interesting features because of narrow boundary and interior layer regions where the solution changes rapidly. Determining the limiting behavior and the location of the internal layers exhibited by the solutions presents both analytical and computational challenges when the boundary value problem is an autonomous problem like (1), (2).

In this paper, we shall give sufficient conditions for the existence of solutions of (1), (2) with either boundary layers or internal layers by assuming that the interval $[A, B]$ contains "turning points" $e_{i}$, where $g\left(e_{i}\right)=0$ with $i=1, \ldots, n$, such that the classical Rankine-Hugoniot condition,

$$
\int_{e_{i}}^{e_{i+1}} g(s) d s=0
$$

fails to determine the location $t_{i}$ of the shock layer which joins the two equilibrium states $u_{L}=e_{i}$ and $u_{R}=e_{i+1}$.

We will also determine the location of the internal layers as a function of the number $n$ of turning points $e_{i} \in[A, B]$, the position of $e_{i} \in[A, B]$, and the order $r_{i}$ of each turning point $e_{i}$. ( $\left(e_{i}\right.$ is a zero of order $r_{i}$ if $g^{(j)}\left(e_{i}\right)=0$ for $j=0, \ldots, r_{i}-1$ and $g^{\left(r_{i}\right)}\left(e_{i}\right) \neq 0$.)

Depending on the order of the turning points, solutions of (1), (2) are shown to exhibit essentially two types of behavior. Turning points of different orders provide

Received June 30, 1993, revised February 4, 1994.

1991 Mathematics Subject Classification: 34E20, 34B10.

Key words and phrases: free layers, singular points, jumps, turning points, singular perturbations. 
solutions with only boundary layers while turning points of the same order provide solutions with internal layers.

Such behavior has been observed by O'Malley $[14,15]$ for Liénard equations. See also Lutz and Goze [10] for equations of the form (1) with $q=0$. Our results extend those of O'Malley and Lutz and Goze since we will show that the behavior of the solutions and the position of shocks (i.e., the standard part of the shock location) remain for all values of $q \in[1,2]$ and for $q=0$ when the Rankine-Hugoniot condition is satisfied. On the contrary, the thickness of the shocks depends on the values of $q$ since it depends only on the type of growth of the equation with $x^{\prime}$ for $x^{\prime} \rightarrow \infty$. The shock region is of order $O\left(\varepsilon^{1 /(2-q)}\right)$ if $q<2$ and of order $O\left(e^{-1 / \varepsilon}\right)$ for the quadratic case $q=2$.

We can find in [1] similar results concerning the fact that the position of a shock does not depend on the type of growth of the equation with $x^{\prime}$ for $x^{\prime} \rightarrow \infty$. In [1] we also determined, for a wide class of equations $\varepsilon x^{\prime \prime}=g(x) f\left(x^{\prime}\right)$, with a Nagumo condition on $f$, the behavior of solutions and the location of shocks when the Rankine-Hugoniot condition is satisfied and $g(x)$ is not zero at the equilibrium states.

Our methods use nonstandard analysis, the observability plane $[5,6]$ and a geometrical study of the singular jumps [2].

In Section 2, we adopt a geometrical approach to study the solution of (1), (2) as a trajectory of the slow-fast system associated with (1) and we describe the jumps in the corresponding observability plane.

In Section 3, we study the trajectory near each turning point in the appropriate phase space of the singular jumps and we give the more important features of these jumps.

In Section 4, we determine the behavior of the solutions and we locate the transition layers. In addition, we give explicit estimates of the first derivative $x^{\prime}(t)$ which are useful to compute numerically the solutions of (1), (2).

Finally, in Section 5 we present several examples including one with a boundary layer due to a higher-order turning point, exhibiting many small deformations caused by other turning points. We also discuss the problems from the numerical point of view.

\section{The boundary value problem viewed in the observability plane}

Let us consider the differential equation (1) as a slow-fast system in the $\left(x, v=x^{\prime}\right)$ phase plane:

$$
\left\{\begin{aligned}
x^{\prime} & =v, \\
\varepsilon v^{\prime} & =-g(x) v^{q} .
\end{aligned}\right.
$$

The solution of the boundary value problem (1), (2) is a trajectory which goes from a point on the vertical line $x=A$ to the vertical line $x=B$ in time $T$.

The slow (or characteristic) curve $\Gamma$ is defined by the vertical lines $x=e$ where $g(e)=0$ if $q=0$ or by the horizontal axis $v=0$ if $q \neq 0$. Thus, for $v=x^{\prime}$ limited, the trajectories either lie near $\Gamma$ or satisfy $x=x_{0}$ where $g\left(x_{0}\right) \neq 0$. The rapid portions of the trajectories (for $v=x^{\prime}$ unlimited), i.e., the jumps, may be observed in the $(x, V)$ observability plane obtained from rescaling the fast variable $v$ by introducing $v=h(V / \varepsilon)$ where $h(\theta)$ is defined by

$$
h \frac{d h}{d \theta}=h^{q}, \quad h(0)=v_{0}, \quad q=0 \text { or } 1 \leq q \leq 2 .
$$




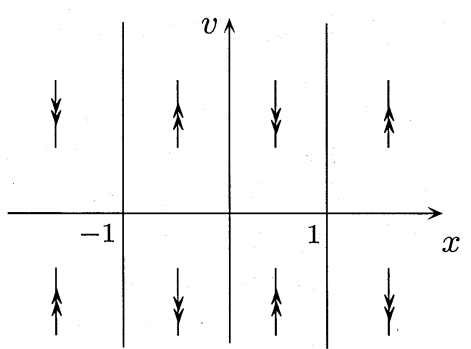

(i)

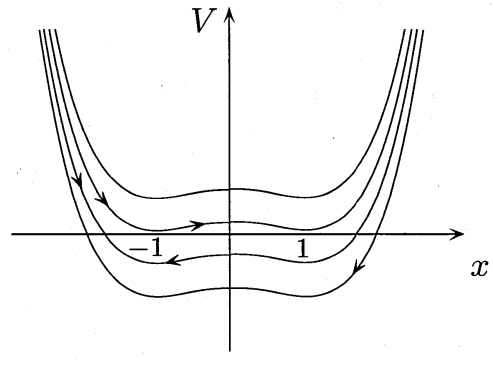

(ii)

Figure 2.1. The trajectories of $\varepsilon x^{\prime \prime}=x\left(x^{2}-1\right) x^{\prime q}$ (i) in the phase space $(x, v)$, (ii) in the observability plane $(x, V)$.

Thus, the rapid trajectories satisfy $d V / d x \simeq-g(x)$ up to an infinitesimal and any jump of (1) is infinitely close to the curve of equation $V=G(x)$ where $G(x)=-\int_{A}^{x} g(s) d s+C$; see Figure 2.1.

We note that, in the $(x, V)$ plane, the jumps are the same for all values of $q$. Only the rescaling $h$ which allows us to study them depends on $q$.

In the $(x, V)$ plane, the slow motions of the trajectories lie near $V=0$. Then any constant portion $e$ of the solution $x(t)$ must satisfy $V(e) \simeq 0$, and we have $G(e)=0$.

Our principal assumption is that the minimum of $G(x)$ in $[A, B]$ (or the maximum of $G(x)$ in $[B, A]$ if $B<A)$ is attained at $n$ values $e_{i}, i=1, \ldots, n$ with $n \geq 1$; see Figure 2.2.

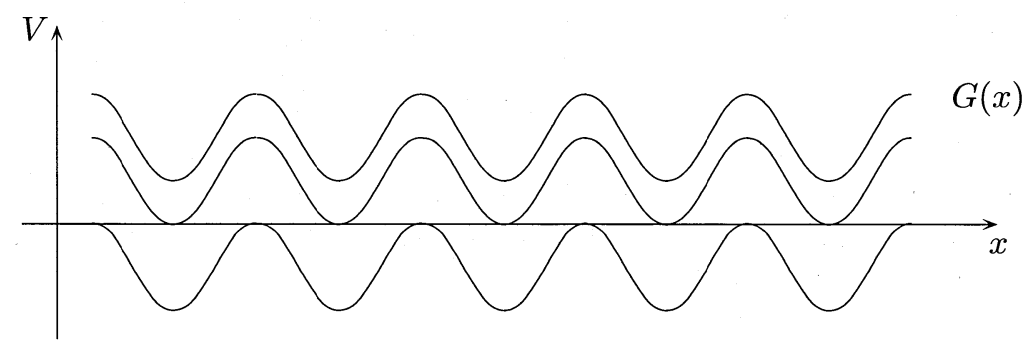

Figure 2.2

We note that by the uniqueness theorem for the initial value problem, no nonconstant solution of (1), (2) can have a zero of $x^{\prime}$ when $1 \leq q \leq 2$. In fact, if $x^{\prime}\left(t^{*}\right)=0$ for some $t^{*}$, the initial value problem with $x\left(t^{*}\right)=M$ and $x^{\prime}\left(t^{*}\right)=0$ would have only the constant solution $x(t) \equiv M$, but this fails in satisfying the two boundary conditions.

Thus, for $1 \leq q \leq 2$ the solution $x(t)$ of the two-point problem (1), (2) must increase monotonically if $A<B$, while we must have $x^{\prime}(t)<0$ if $B<A$. We shall only consider the case $A<B$, since the other follows analogously.

Then any increasing solution to our two-point problem for $1 \leq q \leq 2$ is a trajectory which joins $A$ to $B$ with increasing jumps. These jumps must lie in the half plane $V>0$ and we must have $G(x) \geq 0$. As each limiting solution is a constant on which 
$G(x)$ must vanish, the minima of $G(x)$ are critical in the case of increasing solutions and the limiting solution will jump between endpoint values and certain turning point values $e_{i}$ in the interval $[A, B]$.

For $q=0$, we observe that any constant portion of the solution of (1), (2) must also have a turning point value $e_{i}$ since the limiting solution must be a zero of both $g(x)$ and $G(x)$. The hypothesis about $G(x)$ ensures that $G(x)$ does not change its sign in $[A, B]$ and so any solution for $q=0$ is also strictly monotone.

An analysis of the trajectory in the $(x, V)$ plane may give us the behavior of the solution as, for example, if $G(x)$ has a minimum value at an unique point $e \in[A, B]$. Then the solution is a trajectory which joins $A$ with $B$ with two boundary layers if $e \in(A, B)$, with a boundary layer at $t=0$ if $e=B$ or with a boundary layer at $t=T$ if $e=A$; see Figure 2.3.
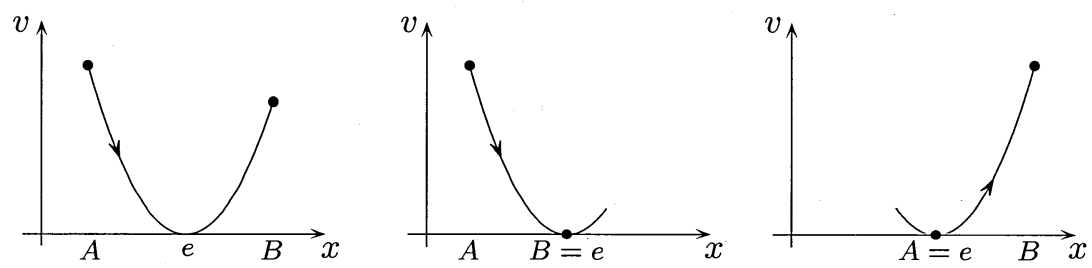

Figure 2.3

However, if $G(x)$ has an equal minimum value at more than one point in $[A, B]$, the analysis in the $(x, V)$ plane does not allow us to determine completely the behavior of the solution.

If, for example, $G(x)$ has the only minimum values at $A$ and $B$, the solution is a trajectory which joins $A$ with $B$ with a jump at some value $t_{0}$. In the $(x, V)$ plane, it must satisfy

$$
V(x(t)) \simeq-\int_{A}^{x} g(s) d s,
$$

and it looks like Figure 2.4, but the jump could be either a boundary layer $\left(t_{0}=\right.$ 0 or $t_{0}=T$ ) or an internal layer. Then, we shall need to perform a more detailed analysis near each turning point.

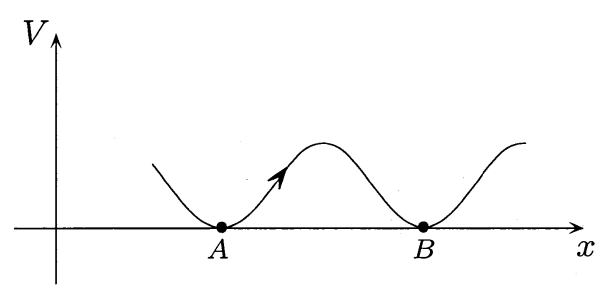

Figure 2.4

The main idea is to consider the inverse function $t(x)$ associated with (1), (2):

$$
\begin{gathered}
\varepsilon t^{\prime \prime}=g(x) t^{\prime 3-q}, \\
t(A)=0, \quad t(B)=T,
\end{gathered}
$$


and to use the properties of the singular jumps of (3). That is, the jumps that take place at the values of $x$ where $g(x)$ vanishes (cf. [2]).

Since the solution $x(t)$ of $(1),(2)$ increases monotonically, the inverse function $t(x)$ exists, is also strictly monotone in $[A, B]$, and satisfies $(3),(4)$. Then if we seek a solution $x(t)$ with a boundary layer at $t=0$ (or at $t=T$ ), $t(x)$ will have a boundary layer at $x=B$ (or at $x=A$ ). If $x(t)$ has an internal layer at $t_{0}$, then $t(x)$ will have two boundary layers connected by the limiting solution $t(x) \simeq t_{0}$. When the boundary conditions $A$ and $B$ are zeros of $g(x)$ the boundary layers exhibited by $t(x)$ are singular jumps for (3). The features of these kinds of jumps play an important part in the description of the behavior of the solution of (1), (2) and in the location of the internal layers.

\section{Turning points and singular jumps}

Let us consider the slow-fast system associated with (3) in the phase space $\left(x, t, u=t^{\prime}\right)$ :

$$
\left\{\begin{aligned}
x^{\prime} & =1, \\
t^{\prime} & =u, \\
\varepsilon u^{\prime} & =g(x) u^{3-q},
\end{aligned}\right.
$$

and assume $e$ is a turning point of order $r$. Then

$$
g(x) \simeq \frac{g^{(r)}(e)(x-e)^{r}}{r !}
$$

near $x=e$.

Depending on the value of $r+q-1$ there is a convenient rescaling of the variables $(x, t, u)$ in order to study the jumps of (5) near $x=e$. In this work, we shall only consider the values of $r$ and $q$ such that $r+q-1>0$. The critical case $r+q-1=0$ which is only possible for $q=0$ with turning points of order $r=1$ (since $r \geq 1$ ) involves a very different and interesting behavior of the singular jumps and it has been studied in a separate work [3]. See also the analytical approaches of Kath, Knessl, and Matkowsky [9] and Ward [17] to determine the positions of the internal layers in the case $r=1$ and $q=0$.

We let

$$
\begin{gathered}
\mathbb{G}(e)=\left\{x: x-e=£ \varepsilon^{1 /(r+q-1)}, £ \text { limited }\right\}, \\
\mathbb{H}(e)=\{x: x \simeq e \text { and } x \notin \mathbb{G}(e)\}, \\
\mathbb{H}^{-}(e)=\mathbb{H}(e) \cap\{x: x<e\}, \\
\mathbb{H}^{+}(e)=\mathbb{H}(e) \cap\{x: x>e\} .
\end{gathered}
$$

Also, $x=\phi \simeq 0$ will indicate an infinitesimal value, $x=£$ a limited value, $x=@$ an appreciable value, and $x \simeq \pm \infty$ an unlimited value.

Definition. We say that the standard value $t_{-}$is the origin of an increasing jump of $t(x)$ in an interval $I$ near $x=e$ if there is $x_{-} \simeq e$ such that $t\left(x_{-}\right) \simeq t_{-}, t^{\prime}\left(x_{-}\right)=£$, and $t^{\prime}(x) \simeq+\infty$ if $t(x) \gg t_{-}$for all $x \in I$.

Analogously, the standard value $t_{+}$is the extremity of the jump if there is $x_{+} \simeq e$ such that $t\left(x_{+}\right) \simeq t_{+}, t^{\prime}\left(x_{+}\right)=£$, and $t^{\prime}(x) \simeq+\infty$ if $t(x) \ll t_{+}$for all $x \in I$. 
We are going to study the trajectories of $(5)$ near $x=e$ in both regions $\mathbb{G}(e)$ and $\mathbb{H}(e)$. By assuming appropriate hypotheses the following proposition states that in the new $(X, T, U)$ phase space defined by

$$
\left\{\begin{array}{l}
X=\frac{x-e}{\varepsilon^{c}}, \\
T=t \\
U=\varepsilon^{c} u,
\end{array}\right.
$$

with $c=1 /(r+q-1)$, the jumps of (5) near $x=e$ behave like the trajectories of

$$
\left\{\begin{array}{l}
\dot{X}=1, \\
\dot{T}=U, \\
\dot{U}=\frac{g^{(r)}(e)}{r !} X^{r} U^{3-q} .
\end{array}\right.
$$

Proposition 3.1. (The behavior in $\mathbb{G}(e)$.) Assume:

(i) $e$ is a zero of $g(x)$ of order $r$ such that $c=1 /(r+q-1)>0$;

(ii) $\gamma(x)$ is a trajectory of (5) with $\gamma\left(x_{0}\right)$ such that $x_{0} \in \mathbb{G}(e), t\left(x_{0}\right)$ and $t^{\prime}\left(x_{0}\right) \varepsilon^{c}$ are limited values, and $\Gamma(X)$ is the image of $\gamma$ by the rescaling (6);

(iii) $\Gamma_{0}(X)$ is a trajectory of $(7)$ with $\Gamma_{0}\left(X_{0}\right) \simeq \Gamma\left(X_{0}\right)$.

Then $\Gamma(X) \simeq \Gamma_{0}(X)$ as long as $\Gamma_{0}(X)$ remains limited. Analogously, $\Gamma(X) \simeq$ $\Gamma_{0}(X)$ as long as $x \in \mathbb{G}(e)$ and $t^{\prime}(x) \varepsilon^{c}$ remains limited.

Proof. The system (5) in the new variables $(X, T, U)$ defined by (6) becomes

$$
\begin{aligned}
\dot{X} & =1, \\
\dot{T} & =U, \\
\dot{U} & =\frac{g\left(e+\varepsilon^{c} X\right)}{\varepsilon^{r c}} U^{3-q} .
\end{aligned}
$$

By hypothesis (i), $\mathbb{S}_{0} \simeq \mathbb{S}$ for all values of $X$ such that $\varepsilon^{c} X \simeq 0$ (i.e., for all $x \simeq e$ ), where $\mathbb{S}_{0}$ and $\mathbb{S}$ are the systems given by (7) and (8), respectively. Consider a trajectory $\gamma(x)$ of (5) with prescribed values at $x_{0} \in \mathbb{G}(e)$. By hypothesis (ii), $\gamma\left(x_{0}\right)$ is, in the new variables, a point $P_{0}\left(X_{0}, T_{0}, U_{0}\right)$ with limited coordinates. Let us consider the trajectory $\Gamma_{0}(X)$ of $\mathbb{S}_{0}$ such that $\Gamma_{0}(X) \simeq P_{0}$. As long as the values of $\Gamma_{0}(X)$ are limited, $\Gamma_{0}(X)$ is contained in the region where the systems $\mathbb{S}$ and $\mathbb{S}_{0}$ are infinitely close. Then by the Short Shadow Lemma [7], we infer that $\Gamma(X)$ exists and is infinitely close to $\Gamma_{0}(X)$ as long as $\Gamma_{0}(X)$ remains limited. Analogously, as long as $x \in \mathbb{G}(e)$ and $t^{\prime}(x) \varepsilon^{c}$ is limited, the values of $\Gamma(X)$ remain limited, so $\Gamma(X)$ is contained in the region where $\mathbb{S} \simeq \mathbb{S}_{0}$. By the Short Shadow Lemma, ${ }^{\circ}(\Gamma(X))$, the shadow of $\Gamma(X)$, is a trajectory, $\Gamma_{0}(X)$, of $\mathbb{S}_{0}$.

Corollary 3.2. Any trajectory $\gamma(x)$ of (5), with $\gamma\left(x_{0}\right)$ such that $x_{0} \in \mathbb{G}(e), t\left(x_{0}\right)$ is limited, and $t^{\prime}\left(x_{0}\right) \varepsilon^{c} \simeq 0$, satisfies $t(x) \simeq t\left(x_{0}\right)$ and $t^{\prime}(x) \varepsilon^{c} \simeq 0$ for all $x \in \mathbb{G}(e)$.

Proof. Consider $\Gamma(X)=(X, T(X), U(X))$, the image of $\gamma(x)$ under (6). Then $\Gamma\left(X_{0}\right)=\left(X_{0}, T_{0}, U_{0}\right)$ with $U_{0}=t^{\prime}\left(x_{0}\right) \varepsilon^{c} \simeq 0$. Consider $\Gamma_{0}(X)$, the trajectory of (7) such that $\Gamma_{0}(X)={ }^{0}\left(\Gamma\left(X_{0}\right)\right)=\left({ }^{0} X_{0},{ }^{0} T_{0}, 0\right)$. Then $\Gamma_{0}(X)=\left(X,{ }^{0} T_{0}, 0\right)$. As $\Gamma_{0}$ remains limited for all $X$ limited, we have, by Proposition 3.1, $\Gamma(X) \simeq \Gamma_{0}(X)$ for all 
$X$ limited. In particular, this implies that $t(x)$ will be almost constant in $\mathbb{G}(e)$ since we must have $t(x)=T(X) \simeq T_{0}=t\left(x_{0}\right)$ and $t^{\prime}(x) \varepsilon^{c}=U(X) \simeq 0$ for all $x \in \mathbb{G}(e)$.

Remark 3.1. The above results determine the minimum order of magnitude of $u=t^{\prime}(x)$ of any jump in $\mathbb{G}(e)$. In fact, any solution that has a jump in $I \subset \mathbb{G}(e)$ must satisfy $t^{\prime}(x) \varepsilon^{c} \gg 0$ for all $x \in \mathbb{G}(e)$.

The next proposition concerns the behavior of the trajectories of $(5)$ in $\mathbb{G}(e)$, for any value of $u(x)$ such that $t^{\prime}(x) \varepsilon^{c} \simeq+\infty$. It states that any trajectory of (5) having prescribed values at some $x_{0} \in \mathbb{G}(e)$ with $t\left(x_{0}\right)$ limited and $t^{\prime}\left(x_{0}\right) \varepsilon^{c} \simeq+\infty$, has an "unbounded" jump in $\mathbb{G}(e)$.

Proposition 3.3. Assume $\gamma(x)$ is a trajectory of (5) with $\gamma\left(x_{0}\right)$ such that $x_{0} \in \mathbb{G}(e)$, $t\left(x_{0}\right)$ is limited, and $t^{\prime}\left(x_{0}\right) \varepsilon^{c} \simeq+\infty$. Then there is an interval $[a, b] \subset \mathbb{G}(e)$ such that $x_{0} \in[a, b], t(a) \simeq-\infty$, and $t(b) \simeq+\infty$.

Proof. Given a trajectory $\gamma(x)$ of $(5)$ with $\gamma\left(x_{0}\right)$ as assumed and such that $u_{0}=t^{\prime}\left(x_{0}\right)$ satisfies $u_{0} \varepsilon^{c} \simeq+\infty$, consider the following rescaling:

$$
\left\{\begin{aligned}
X & =(x-e) u_{0}, \\
T & =t \\
W & =u / u_{0} .
\end{aligned}\right.
$$

The system (5) is then transformed into

$$
\left\{\begin{aligned}
\dot{X} & =1 \\
\dot{T} & =W \\
\dot{W} & =\left(u_{0}^{q-1} \varepsilon\right)^{-1} g\left(e+\frac{X}{u_{0}}\right) W^{3-q} .
\end{aligned}\right.
$$

As $x=e$ is a zero of $g$ of order $r$, we can rewrite the third component of (10) near $x=e$ as

$$
\dot{W}=\left(u_{0} \varepsilon^{c}\right)^{-1 / c} \frac{g^{(r)}(e)}{r !}(1+\phi) X^{r} W^{3-q}, \quad \phi \simeq 0 .
$$

As $u_{0} \varepsilon^{c} \simeq+\infty$, this system is infinitely close to

$$
\left\{\begin{array}{c}
\dot{X}=1, \\
\dot{T}=W, \\
\dot{W}=0,
\end{array}\right.
$$

for all $(X, T, W)$ limited. Let $\Gamma(X)$ be the image of $\gamma(x)$ by $(9)$ where $\Gamma\left(X_{0}\right)=$ $\left(X_{0}, T_{0}, 1\right)$ is the point $\gamma\left(x_{0}\right)$ in the new variables.

If $\Gamma_{0}(X)$ is the trajectory of (11) such that $\Gamma_{0}\left(X_{0}\right)=\Gamma\left(X_{0}\right)$, then $\Gamma_{0}(X)=$ $\left(X, X+T_{0}-X_{0}, 1\right)$. As $\Gamma_{0}(X)$ remains limited for all $X$ limited, we infer from the Short Shadow Lemma that $\Gamma(X)$ exists and $\Gamma(X) \simeq \Gamma_{0}(X)$ for these values of $X$. By permanence, there is a value $\omega \simeq+\infty$ such that $\Gamma(X) \simeq \Gamma_{0}(X)$ for all $|X| \leq \omega$. As we can take $\omega$ such that $\omega / u_{0}=£$ and $\omega / u_{0}>x_{0}$, we have found an interval $[a, b]=\left[e-\omega / u_{0}, e+\omega / u_{0}\right] \subset \mathbb{G}(e)$ where $t(x)=T(X) \simeq X+T_{0}-X_{0}$, and we have $t(a)=-t(b)$ and $t(b) \simeq+\omega+T_{0}-X_{0} \simeq+\infty$, since $T_{0}$ and $X_{0}$ are limited.

Finally, we are interested in the behavior of trajectories of $(5)$ in $\mathbb{H}(e)$. We will show that no jump is possible in $\mathbb{H}(e)$ when the velocity $u(x)$ is $O\left(\varepsilon^{c}\right)$. 
Proposition 3.4. (The behavior in $\mathbb{H}(e)$.) If $\gamma(x)$ is a trajectory of (5) with $\gamma\left(x_{0}\right)$ such that $x_{0} \in \mathbb{H}(e)$ and both $t\left(x_{0}\right)$ and $t^{\prime}\left(x_{0}\right) \varepsilon^{c}$ are limited, then for $x \in \mathbb{H}(e)$, $t(x) \simeq t\left(x_{0}\right)$ as long as $t^{\prime}\left(x_{0}\right) \varepsilon^{c}$ is limited.

Proof. Let us introduce the new variables $z=(x-e)^{r+1} \varepsilon^{-c(r+1)} /(r+1)$ and $U=u \varepsilon^{c}$. Then the system (5) becomes

$$
\left\{\begin{array}{l}
\dot{x}=\varepsilon^{c} \frac{\varepsilon^{r c}}{(x-e)^{r}}, \\
\dot{t}=U \frac{\varepsilon^{r c}}{(x-e)^{r}}, \\
\dot{U}=\frac{g^{(r)}(e)}{r !} U^{3-q}(1+\phi),
\end{array}\right.
$$

for $x$ near $x=e$, where $\dot{x}=\frac{d}{d z}$ and $\phi \simeq 0$. As $(x-e) / \varepsilon^{c} \simeq \infty$ for all $x \in \mathbb{H}(e),(12)$ is infinitely close to

$$
\begin{aligned}
\dot{x} & =0 \\
\dot{t} & =0 \\
\dot{U} & =\frac{g^{(r)}(e)}{r !} U^{3-q}
\end{aligned}
$$

as long as $U$ remains limited and $x \in \mathbb{H}(e)$. Then, by the Short Shadow Lemma, any trajectory $\gamma(x)$ of $(5)$ is, in the new variables, infinitely close to a trajectory $\Gamma_{0}$ of (13), where $\Gamma_{0}$ satisfies $x \simeq x_{0}$ and $t(x) \simeq t\left(x_{0}\right)$ as long as $t^{\prime}(x) \varepsilon^{c}$ remains limited for $x \in \mathbb{H}(e)$.

Remark 3.2. The above results describe the general behavior of the fast trajectories in both regions $\mathbb{G}(e)$ and $\mathbb{H}(e)$. In particular, we are interested in solutions of (5) which pass through some limited point $\left(x_{0}, t\left(x_{0}\right)\right)$ with $x_{0} \in \mathbb{G}(e)$ and which exhibit a jump near $x=e$ with an origin $t_{-}$or an extremity $t_{+}$(or both). In this case, precise estimates of $t(x)$ and $t^{\prime}(x)$ may be given by means of the trajectories $\Gamma_{0}$ of system (7) in some appropriate interval, as we will show in the next theorem. Moreover, the origin $t_{-}$and the extremity $t_{+}$may be calculated as a function of $x_{0}, t\left(x_{0}\right), g^{(r)}(e)$, $\varepsilon, t^{\prime}\left(x_{0}\right), r$, and $q$.

We note that such a trajectory $\Gamma_{0}$ which passes through $\left(X_{0}, T_{0}, U_{0}\right)$ is given by

$$
\Gamma_{0}(X)=\left(X_{0}, T_{0}+K\left(X, k_{0}\right), H\left(X, k_{0}\right)\right)
$$

where $K\left(X, k_{0}\right)=\int_{X_{0}}^{X} H\left(s, k_{0}\right) d s, k_{0}$ is a constant value such that $U_{0}=H\left(X_{0}, k_{0}\right)$, and

$$
H\left(X, k_{0}\right)= \begin{cases}\exp \left(k_{0}\right) \exp \left(d_{e} X^{r+1}\right) & \text { if } q=2 \\ \left(k_{0}-(2-q) d_{e} X^{r+1}\right)^{-1 /(2-q)} & \text { if } q<2\end{cases}
$$


The function $K$ may be written as follows:

$$
K\left(X, k_{0}\right)=\left\{\begin{array}{lr}
\exp \left(k_{0}\right) \int_{X_{0}}^{X} \exp \left(d_{e} \tau^{r+1}\right) d \tau, & \text { if } q=2 \\
\left|k_{0}\right|^{1 / c(r+1)(q-2)} \int \frac{x_{0}}{\left|k_{0}\right|^{1 /(r+1)}}\left(\sigma-(2-q) d_{e} \tau^{r+1}\right)^{-1 /(2-q)} d \tau, & \text { if } q<2
\end{array}\right.
$$

where

$$
k_{0}= \begin{cases}\operatorname{Ln}\left|\varepsilon^{c} t^{\prime}\left(x_{0}\right)\right|-d_{e} X_{0}^{r+1} & \text { if } q=2, \\ \left(\varepsilon^{c} t^{\prime}\left(x_{0}\right)\right)^{q-2}+(2-q) d_{e} X_{0}^{r+1} & \text { if } q<2\end{cases}
$$

$d_{e}=g^{(r)}(e) /(r+1) !$, and $\sigma=k_{0} /\left|k_{0}\right|$.

Theorem 3.5. (Origin and extremity of a singular jump.) Assume $t(x)$ is a solution of (3), i.e., $\varepsilon t^{\prime \prime}=g(x) t^{t^{3-q}}$, such that $t\left(x_{0}\right)$ is limited with $x_{0} \in \mathbb{G}(e)$, and that $x=e$ is a zero of $g(x)$ of order $r$ such that $c=1 /(r+q-1)>0$.

(1) If $t^{\prime}\left(x_{0}\right) \varepsilon^{c}=@^{+}$, there is an interval $I \subset \mathbb{G}(e)$ such that $t(x)$ has an increasing jump in $I$ and $t^{\prime}(x) \varepsilon^{c}=@^{+}$for all $x \in I$. Moreover, $t^{\prime}(x) \varepsilon^{c} \simeq H\left(X, k_{0}\right)$ and $t(x) \simeq t\left(x_{0}\right)+K\left(X, k_{0}\right)$ in $I$, where $H$ and $K$ are the functions given by (15) and (16) and $k_{0}$ is an appreciable constant given by (17).

In addition, if $H\left(X, k_{0}\right)$ exists in $\left(-\infty, X_{0}\right]$ and $\lim _{X \rightarrow-\infty} H\left(X, k_{0}\right)=0$, then there is an $x_{-} \in \mathbb{H}^{-}(e)$ such that $t^{\prime}(x) \varepsilon^{c} \simeq H\left(X, k_{0}\right), t(x) \simeq t\left(x_{0}\right)+K\left(X, k_{0}\right)$ in $\left[x_{-}, x_{0}\right]$, $t^{\prime}(x) \varepsilon^{c}=@$ in $\left[x_{-}, x_{0}\right] \cap \mathbb{G}(e)$, and the jump has an origin $t_{-} \simeq t\left(x_{-}\right)$where

$$
t_{-}=t\left(x_{0}\right)+\lim _{X \rightarrow-\infty} K\left(X, k_{0}\right)+\phi, \quad \text { with } \phi \simeq 0 .
$$

Analogously, if $H\left(X, k_{0}\right)$ exists in $\left[X_{0},+\infty\right]$ and $\lim _{X \rightarrow+\infty} H\left(X, k_{0}\right)=0$, there is an $x_{+} \in \mathbb{H}^{+}(e)$ such that $t^{\prime}(x) \varepsilon^{c} \simeq H\left(X, k_{0}\right), t(x) \simeq t\left(x_{0}\right)+K\left(X, k_{0}\right)$ in $\left[x_{0}, x_{+}\right]$, $t^{\prime}(x) \varepsilon^{c}=@$ in $\left[x_{0}, x_{+}\right] \cap \mathbb{G}(e)$, and the jump has an extremity $t_{+} \simeq t\left(x_{+}\right)$where

$$
t_{+}=t\left(x_{0}\right)+\lim _{X \rightarrow+\infty} K\left(X, k_{0}\right)+\phi, \quad \text { with } \phi \simeq 0 .
$$

(2) If the solution $t(x)$ has an increasing jump near $x=e$ with an origin $t_{-} \ll t\left(x_{0}\right)$ (or an extremity $t_{+} \gg t\left(x_{0}\right)$ ), then $t^{\prime}(x) \varepsilon^{c}$ is appreciable, $t^{\prime}(x) \varepsilon^{c} \simeq H\left(X, k_{0}\right)$, and $t(x) \simeq t\left(x_{0}\right)+K\left(X, k_{0}\right)$ for $x \in \mathbb{G}(e), x \leq x_{0}\left(\right.$ for $\left.x \in \mathbb{G}(e), x \geq x_{0}\right)$. In addition, $H\left(X, k_{0}\right)$ exists in $\left(-\infty, X_{0}\right]$ with $\lim _{X \rightarrow-\infty} H\left(X, k_{0}\right)=0$ in the case of the origin, and $H\left(X, k_{0}\right)$ exists in $\left[X_{0},+\infty\right]$ with $\lim _{X \rightarrow+\infty} H\left(X, k_{0}\right)=0$ in the case of extremity.

Proof. We shall only prove the theorem in the case of a jump with an origin $t_{-}$. The proof in the case of an extremity $t_{+}$follows analogously.

(1) Consider a solution $\gamma(x)=(x, t(x), u(x))$ of the initial value problem (5) associated with (3), where $\gamma\left(x_{0}\right)=\left(x_{0}, t_{0}, u_{0}\right)$ such that $x_{0} \in \mathbb{G}(e)$ and $t_{0}=t\left(x_{0}\right)$ is limited. Assume $\Gamma(X)$ is the image of $\gamma(x)$ by the rescaling $(6)$ and consider $\gamma_{0}(X)$, the trajectory of $(7)$ such that $\Gamma_{0}\left(X_{0}\right)=\Gamma\left(X_{0}\right)$. Then $\Gamma_{0}(X)=$ $\left(X, T_{0}+K\left(X, k_{0}\right), H\left(X, k_{0}\right)\right)$, where the functions $H$ and $K$ and the constant $k_{0}$ are given by $(15),(16)$, and (17), respectively. As $\Gamma\left(X_{0}\right)=\left(X_{0}, T_{0}, U_{0}\right)$ is limited and $U_{0}=t^{\prime}\left(x_{0}\right) \varepsilon^{c}=@, k_{0}$ is appreciable if $q<2$ or limited if $q=2$ and $H$ is an almost standard function. As $H$ is continuous in some interval of $X$ limited and $H\left(X_{0}, k_{0}\right)=@$, there are $X_{1}$ and $X_{2}$ limited with $X_{1} \ll X_{2}$ such that $H\left(X, k_{0}\right)=@$ 
in $\left[X_{1}, X_{2}\right]$. Thus, $\Gamma_{0}(X)$ remains limited in $\left[X_{1}, X_{2}\right]$ and, by Proposition 3.1, $\Gamma(X)$ exists and satisfies $\Gamma(X) \simeq \Gamma_{0}(X)$ for all $X \in\left[X_{1}, X_{2}\right]$. Therefore, there is an interval $I=\left[x_{1}, x_{2}\right] \subset \mathbb{G}(e)$ such that $t^{\prime}(x) \varepsilon^{c} \simeq H\left(X, k_{0}\right)$ and $t(x) \simeq T_{0}+\int_{X_{1}}^{X} H\left(s, k_{0}\right) d s$ for all $x \in I$; the solution has a jump in $I$ since

$$
t\left(x_{2}\right)-t\left(x_{1}\right) \simeq \int_{X_{1}}^{X_{2}} H\left(s, k_{0}\right) d s \simeq @\left(X_{2}-X_{1}\right)=@
$$

where $X_{2}-X_{1}=@$. In addition, if $H\left(X, k_{0}\right)$ exists in $\left(-\infty, X_{0}\right], \lim _{X \rightarrow-\infty} H\left(X, k_{0}\right)$ $=0$, and $\Gamma_{0}(X)$ remains limited for all $X$ limited, $X \leq X_{0}$, then we have $\Gamma(X) \simeq$ $\Gamma_{0}(X)$ for those values of $X$. By permanence, there is $\omega_{-} \simeq-\infty$ (that we can choose so that $\left.\omega_{-} \varepsilon^{c} \simeq 0\right)$ such that $\Gamma(X) \simeq \Gamma_{0}(X)$ for all $X \in\left[\omega_{-}, X_{0}\right]$. Then there is $x_{\omega}=e+\omega_{-} \varepsilon^{c} \in \mathbb{H}^{-}(e)$ such that $t^{\prime}(x) \varepsilon^{c} \simeq H\left(X, k_{0}\right)$ and $t(x) \simeq t\left(x_{0}\right)+K\left(X, k_{0}\right)$ for all $x \in\left[x_{\omega}, x_{0}\right]$. Moreover, $t^{\prime}(x) \varepsilon^{c}=@$ for all $x \in\left[x_{\omega}, x_{0}\right] \cap \mathbb{G}(e)$ and $t^{\prime}(x) \varepsilon^{c} \simeq 0$ for all $x \in\left[x_{\omega}, x_{0}\right] \cap \mathbb{H}^{-}(e)$. Since $\lim _{X \rightarrow-\infty} H\left(X, k_{0}\right)=0$, we have $\left(g^{(r)}(e) X^{r+1}\right)<0$ for $X<0$ (see (15)). Also, for $x$ near $e, \varepsilon u^{\prime}(x)=g^{(r)}(e)(x-e)^{r}(1+\phi) / r !, \phi \simeq 0$, so $t^{\prime}(x)$ increases for $x \simeq e, x \leq e$. Then $t^{\prime}(x) \varepsilon^{c} \simeq 0$ for all $x \in \mathbb{H}^{-}(e)$, and, by Proposition $3.4, t(x)$ is almost constant in $H^{-}(e)$, so we have

$$
t(x) \simeq t\left(x_{\omega}\right) \simeq T_{0}+\int_{X_{0}}^{\omega} H\left(s, k_{0}\right) d s
$$

for all $x \in \mathbb{H}^{-}(e)$.

If $x_{-} \in \mathbb{H}^{-}(e), x_{-} \leq x_{\omega}$ such that $t^{\prime}\left(x_{-}\right)=£$, then $t\left(x_{-}\right) \simeq t\left(x_{\omega}\right)$. As $\left|H\left(X, k_{0}\right)\right| \leq{ }^{0} H\left(X, k_{0}^{\prime}\right)$ in $\left(-\infty, X_{0}\right]$ for some standard value $k_{0}^{\prime}$ and since, for any $k_{0}^{\prime},{ }^{0} H\left(X, k_{0}^{\prime}\right)$ is a standard integrable function for all $X \in\left(-\infty, X_{0}\right], X_{0}$ limited, we have, by the lemma of dominated approximation [16],

$$
K\left(\omega, k_{0}\right)=\int_{X_{0}}^{\omega} H\left(s, k_{0}\right) d s \simeq \int_{X_{0}}^{-\infty} H\left(s, k_{0}\right) d s=\lim _{X \rightarrow-\infty} K\left(X, k_{0}\right) .
$$

Then $t\left(x_{\omega}\right) \simeq t\left(x_{0}\right)+\lim _{X \rightarrow-\infty} K\left(X, k_{0}\right)$, and $t_{-}={ }^{0}\left(t\left(x_{-}\right)\right)$is the origin of the jump. In fact, if $t\left(x_{0}\right) \geq t(x) \gg t_{-}$, we must have $X \in\left[\omega, X_{0}\right]$ and $X$ must be limited. As $t^{\prime}(x) \varepsilon^{c}=$ @ for this value, we have $t^{\prime}(x) \simeq+\infty$.

(2) Assume now that $t(x)$ is a solution of (3) with an increasing jump in an interval $I$ near $x=e$ and $t\left(x_{0}\right)$ is a limited value with $x_{0} \in \mathbb{G}(e)$. As the jump has an origin $t_{-}$, there is $x_{-} \simeq e$ such that $t\left(x_{-}\right) \simeq t_{-}, t^{\prime}\left(x_{-}\right)=£, £$ limited positive and $t^{\prime}(x) \simeq+\infty$ if $t(x) \gg t_{-}$. Then we note that $u(x)=t^{\prime}(x)$ must increase in some interval contained in $\left[x_{-}, e\right]$. As $\operatorname{sign}\left(u^{\prime}(x)\right)=\operatorname{sign}(g(x))$ because $\varepsilon u^{\prime}(x)=g(x) u^{3-q}$, and since $e$ is a zero of $g(x)$ of order $r, g(x)=g^{(r)}(e)(x-e)^{r}(1+\phi) / r$ ! for $x$ near $e$. Then we must have either $g^{(r)}(e)<0$ if $r$ is an odd integer or $g^{(r)}(e)>0$ if $r$ is an even integer, and, actually, $u(x)$ increases for all $x$ near $e$ and $x<e$.

Now we will show that $x_{-} \in \mathbb{H}^{-}(e)$. As $t_{-} \ll t\left(x_{0}\right)$ and the jump increases, $x_{-}<x_{0}$ and we must have $x_{-} \in \mathbb{H}^{-}(e)$. Otherwise, if $x_{-} \in \mathbb{G}(e)$, we would have $t^{\prime}\left(x_{-}\right) \varepsilon^{c}=£ \varepsilon^{c} \simeq 0$. Then, by Corollary 3.2 , the solution would be almost constant for all $x \in \mathbb{G}(e)$, and we would have $t_{-} \simeq t\left(x_{0}\right)$, which is absurd.

Now let us prove that $t^{\prime}(x) \varepsilon^{c}=@$ with @ appreciable in $\left[x_{-}, x_{0}\right] \cap \mathbb{G}(e)$. Suppose first that $t^{\prime}\left(x_{1}\right) \varepsilon^{c} \simeq 0$ for some $x_{1} \in \mathbb{G}(e), x_{1}<x_{0}$. Then, by Corollary 3.2, $t(x)$ would be almost constant in $\mathbb{G}(e)$ and $t^{\prime}(x) \varepsilon^{c} \simeq 0$ for all $x \in \mathbb{G}(e)$. This implies $I \subset \mathbb{H}(e)$ since $t(x)$ has a jump in $I$. Moreover, $I \subset \mathbb{H}^{-}(e)$, since the increasing jump has an origin $t_{-} \ll t\left(x_{0}\right)$. Then, by Proposition 3.4, we must have $t^{\prime}(x) \varepsilon^{c} \simeq+\infty$ for $x \in I$. 
As $t^{\prime}(x)$ increases near $x=e$ for $x<e$, there would be $x_{2} \in \mathbb{G}(e), x<x_{2} \leq x_{0}$ for some $x \in I \subset \mathbb{H}^{-}$such that $t^{\prime}\left(x_{2}\right) \varepsilon^{c} \simeq+\infty$, which is absurd.

Suppose now $t^{\prime}\left(x_{1}\right) \varepsilon^{c} \simeq+\infty$ for some $x_{1} \in \mathbb{G}(e), x_{1}<x_{0}$. Then, by Proposition 3.3 , there is $a<x_{0}, a \in \mathbb{G}(e)$, such that $t\left(x_{0}\right)-t(a) \simeq+\infty$, which is not possible since $0<t\left(x_{0}\right)-t(a)<t\left(x_{0}\right)-t\left(x_{-}\right) \simeq t\left(x_{0}\right)-t_{-}$and $t\left(x_{0}\right)$ and $t_{-}$are limited values. This proves that $t^{\prime}(x) \varepsilon^{c}=@$ with @ appreciable in $\left[x_{-}, x_{0}\right] \cap \mathbb{G}(e)$.

Consider $\Gamma(X)=(X, T(X), U(X))$ the image by (6) of the trajectory $\gamma(x)$ where $\gamma\left(x_{0}\right)=\left(x_{0}, t\left(x_{0}\right), t^{\prime}\left(x_{0}\right)\right)$. Since $U(X)=t^{\prime}(x) \varepsilon^{c}=@$ in $\left[x_{-}, x_{0}\right] \cap \mathbb{G}(e), \Gamma(X)$ remains limited for all $X$ limited in $\left[X_{-}, X_{0}\right]$, where $X_{-} \simeq-\infty$ because $x_{-} \in H^{-}(e)$. Then it follows from Proposition 3.1 that $\Gamma(X) \simeq \Gamma_{0}(X)$ for those values of $X$, where $\Gamma_{0}(X)$ is the trajectory of $(7)$ given by (14) with $\Gamma_{0}\left(X_{0}\right)=\Gamma\left(X_{0}\right)=\left(X_{0}, T_{0}, U_{0}\right)$. By permanence, there is $\omega \leq x_{-}, \omega \simeq-\infty$, such that $\Gamma(X) \simeq \Gamma_{0}(X)$ for all $X \in\left[\omega, X_{0}\right]$, and we have $U(X)=t^{\prime}(x) \varepsilon^{c} \simeq H\left(X, k_{0}\right)$ for all $X \in\left[\omega, X_{0}\right]$. Then $H\left(X, k_{0}\right)$ exists at least in $\left[\omega, X_{0}\right]$, and as $\operatorname{sign}\left(g^{(r)}(e) X^{r+1}\right)<0$ for $X<0, H\left(X, K_{0}\right)$ is defined in $\left(-\infty, X_{0}\right]$ and $\lim _{X \rightarrow-\infty} H\left(X, k_{0}\right)=0$.

Remark 3.3. Theorem 3.5 gives the origin $t_{-}$and the extremity $t_{+}$of a singular jump near $x=e$ exhibited by a solution $t(x)$ of (4) as a function of $x_{0}, t\left(x_{0}\right), t^{\prime}\left(x_{0}\right), g^{(r)}(e)$, $\varepsilon, r$, and $q$ when $x_{0} \in \mathbb{G}(e)$. In the special case $x_{0}=e$ (i.e., $X_{0}=0$ ), the formulas (18) and (19) concerning the extremities of the jump may be written as follows:

$$
\begin{aligned}
& t_{-}=t(e)-\alpha_{e}\left(t^{\prime}(e) \varepsilon^{1 /(r+q-1)}\right)^{(r+q-1) /(r+1)} C(r, q)+\phi \\
& t_{+}=t(e)+\alpha_{e}\left(t^{\prime}(e) \varepsilon^{1 /(r+q-1)}\right)^{(r+q-1) /(r+1)} C(r, q)+\phi
\end{aligned}
$$

where $\phi \simeq 0$ and

$$
C(r, q)= \begin{cases}\frac{(r !)^{1 /(r+1)}}{(r+1)^{r /(r+1)}} \Gamma\left(\frac{1}{r+1}\right) & \text { if } q=2, \\ \frac{(r !)^{1 /(r+1)}}{\left((2-q)(r+1)^{r}\right)^{1 /(r+1)}} B\left(\frac{1}{r+1}, \frac{r+q-1}{(2-q)(r+1)}\right) & \text { if } q<2 .\end{cases}
$$

The constant $\alpha_{e}=1 /\left|g^{(r)}(e)\right|^{1 /(r+1)}$, and $\Gamma$ and $B$ are the gamma and beta functions.

Corollary 3.6. Assume $t(x)$ is the solution of the initial problem (3) with $t(e)$ and $t^{\prime}(e)$ prescribed and $e$ is a zero of $g(x)$ or order $r$ such that $c=1 /(r+q-1)>0$.

If $t(e)$ is limited and $t^{\prime}(e) \varepsilon^{c}=@+$, then the solution has an increasing jump near $x=e$. In addition,

(1) If $e$ is a minimum point of $G(x)$, then there is an interval $I=\left[x_{-}, x_{+}\right]$, $x_{-} \in H^{-}(e), x_{+} \in \mathbb{H}^{+}(e)$ such that $t(x)$ has a jump in $I$ with an origin $t_{-}$and an extremity $t_{+}$and $t_{-} \simeq t\left(x_{-}\right) \ll t(e) \ll t\left(x_{+}\right) \simeq t_{+}$.

(2) If $e$ is an inflection point of $G(x)$ such that $g^{(r)}(e)<0$, then there is an interval $I=\left[e, x_{+}\right], x_{+} \in \mathbb{H}^{+}(e)$ such that $t(x)$ has a jump in $I$ with an extremity $t_{+}$and $t(e) \ll t\left(x_{+}\right) \simeq t_{+}$. The opposite conclusion follows if $e$ is an inflection point of $G(x)$ such that $g^{(r)}(e)>0$.

The values of $t_{-}$and $t_{+}$are given by (20) and (21).

Proof. By Theorem 3.5(1), the solution $t(x)$ has a jump in some interval $I^{\prime} \subset \mathbb{G}(e)$ and $t^{\prime}(x) \varepsilon^{c} \simeq H\left(X, k_{0}\right)$ for all $x \in I^{\prime}$ where $H$ and $k_{0}$ are given by (15), (17) and $e$ is a zero of $g(x)$ of order $r$. 
(1) If $e$ is a minimum point of $G(x)$, then $g^{(r)}(e)<0$ with $r$ an odd integer. Then $H\left(X, k_{0}\right)$ exists in $(-\infty,+\infty)$ and $\lim _{X \rightarrow \pm \infty} H\left(X, k_{0}\right)=0$, so by Theorem $3.5(2)$ we have the stated conclusion.

(2) If $e$ is an inflection point of $G(x)$ such that $g^{(r)}(e)<0$ where $r$ is now an even integer, then $H\left(X, k_{0}\right)$ exists in $[0,+\infty)$ and $\lim _{X \rightarrow+\infty} H\left(X, k_{0}\right)=0$, so the conclusion also follows from Theorem 3.5(1).

Corollary 3.7. Assume $t(x)$ is a solution of (3) that has a jump near $x=e$ and $e$ is a zero of $g(x)$ of order $r$ such that $r+q-1>0$.

If the jump has an origin $t_{-}$and an extremity $t_{+}$such that $t \ll t(e) \ll t_{+}$, then $t^{\prime}(x) \varepsilon^{1 /(r+q-1)}=@$, @ appreciable, for all $x \in \mathbb{G}(e)$.

In addition,

$$
\begin{aligned}
& t_{+}-t_{-}=2 \alpha_{e}\left(t^{\prime}(e) \varepsilon^{1 /(r+q-1)}\right)^{(r+q-1) /(r+1)} C(r, q)+\phi, \\
& t_{+}+t_{-}=2 t(e)+\phi
\end{aligned}
$$

where $\phi \simeq 0, C(r, q)$ is given by $(22)$, and $\alpha_{e}=1 /\left|g^{(r)}(e)\right|^{1 /(r+1)}$.

Proof. As $t_{-} \ll t(e) \ll t_{+}$, it follows from Theorem 3.5(2) that $t^{\prime}(x) \varepsilon^{c}=$ @, @ appreciable, for all $x=\mathbb{G}(e)$ with $c=1 /(r+q-1)$, and the corresponding function $H\left(X, k_{0}\right)$ exists in $(-\infty,+\infty)$ and satisfies $\lim _{X \rightarrow \pm \infty} H\left(X, k_{0}\right)=0$. Then, by Theorem 3.5(1), the extremities of the jump satisfy

$$
\begin{aligned}
& t_{-}=t(e)+\int_{0}^{-\infty} H\left(s, k_{0}\right) d s+\phi \\
& t_{+}=t(e)+\int_{0}^{+\infty} H\left(s, k_{0}\right) d s+\phi .
\end{aligned}
$$

As, in this case, $H$ is an even function, the stated relationships follow easily.

Corollary 3.8. The thickness of any jump near $x=e$ with an origin and an extremity is $\mathbb{G}(e)$.

Remark 3.4. Relationships (20), (21) are useful in different ways. They provide the extremities of a jump of the solution $\gamma(x)$ of the initial value problem (5) when $\gamma(e)$ is prescribed. Conversely, suppose a pair of values $\left(t_{-}, t_{+}\right)$are given and we look for a solution with a jump having $t_{-}$as origin and $t_{+}$as extremity. Then, by means of (20), (21), we can determine (up to an infinitesimal) the initial conditions $t(e), t^{\prime}(e)$ in order to get such a solution. This is useful in computing numerically the solutions of the boundary value problem (1), (2) (see Section 4). The order of magnitude of $t^{\prime}(x)$ during the jump, the thickness $\left(O\left(\varepsilon^{1 / r+q-1}\right)\right.$ for bounded jumps), and the relationships (20) and (21) will allow us to determine the behavior of the solution $x(t)$ and the location of the transition layers.

\section{Boundary and internal layers}

4.1. The position of the shocks. We consider again the boundary value problem $P_{q}$ :

$$
\begin{gathered}
\varepsilon x^{\prime \prime}+g(x) x^{\prime q}=0, \quad 0<t<T, \\
x(0)=A, \quad x(T)=B,
\end{gathered}
$$


where $q=0$ or $1 \leq q \leq 2$ and $T, A$, and $B$ are standard values.

The principal result of this section is the following.

Theorem 4.1. Assume

(i) The minimum of $G(x)=-\int_{A}^{x} g(s) d s$ in $[A, B]$ is attained only at $A$ and $B$;

(ii) $A$ and $B$ are zeros of $g(x)$ of orders $r_{A}$ and $r_{B}$, respectively, such that $r_{A}+q-1>0$ and $r_{B}+q-1>0$.

(1) If $r_{A}=r_{B}$, the solution of $P_{q}$ has an internal layer at

$$
t_{0}=T\left[1+\frac{\alpha_{B}}{\alpha_{A}}\right]^{-1},
$$

where $\alpha_{e}=1 /\left|g^{(r)}(e)\right|^{1 /(r+1)}$ for $e=A, B$, and $r=r_{A}=r_{B}$.

(2) If $r_{A}<r_{B}\left(\right.$ or $\left.r_{A}>r_{B}\right)$, the solution of $P_{q}$ has a boundary layer at $t_{0}=0$ (or at $\left.t_{0}=T\right)$.

In addition, the solution $x(t)$ of $P_{q}$ satisfies

$$
x^{\prime}(T)=x^{\prime}(0)=\varepsilon^{1 /(r+q-1)}(\alpha C(r, q))^{(r+1) /(r+q-1)}(1+\phi),
$$

where $r=\max \left\{r_{A}, r_{B}\right\}$ and $\alpha=\alpha_{A} / t_{0}$ if $r=r_{A}=r_{B}, \alpha=\alpha_{B} / T$ if $r=r_{B}>r_{A}$, or $\alpha=\alpha_{A} / T$ if $r=r_{A}>r_{B}, \phi \simeq 0$, and $C(r, q)$ is given by $(22)$.

Proof. (1) Let us first consider the case $r_{A}=r_{B}=r$. If we suppose there exists a boundary layer at $t_{0}=0$, the inverse function $t(x)$ of the solution $x(t)$ of $P_{q}$ has a boundary layer at $e=B$ and $t(x)$ satisfies $t(x) \simeq 0$ for $x \simeq A, x \geq A$. This singular jump at $x=B$ has $t_{-}=0$ as origin, hence, by Theorem $3.5(2), t^{\prime}(B) \varepsilon^{1 /(r+q-1)}=@$. Since $G(B)=0$ because of assumption (i), $t^{\prime}(A)=t^{\prime}(B)$, and since $r_{A}=r_{B}$, we have $t^{\prime}(A) \varepsilon^{1 /(r+q-1)}=@$. Then, by Corollary 3.6, the solution also has a jump in at least some interval $\left[A, x_{+}\right], x_{+} \simeq A$, which is absurd since $t(x) \simeq 0$ for $x \simeq A$ and $x \leq A$. In a similar way we prove that a boundary layer at $t_{0}=T$ is not possible.

Then, if $r_{A}=r_{B}=r$, the solution $x(t)$ of $P_{q}$ has an internal layer at $t_{0}$ and the inverse $t(x)$ has two boundary layers. The singular jump at $x=A$ has $t_{+}=t_{0}$ as the extremity, so we have

$$
t_{0}=t(A)+\alpha_{A}\left(t^{\prime}(A) \varepsilon^{1 /(r+q-1)}\right)^{(r+q-1) /(r+1)} C(r, q)+\phi
$$

while the singular jump at $x=B$ has $t_{-}=t_{0}$ as the origin, so

$$
t_{0}=t(B)-\alpha_{B}\left(t^{\prime}(B) \varepsilon^{1 /(r+q-1)}\right)^{(r+q-1) /(r+1)} C(r, q)+\phi, \quad \phi \simeq 0 .
$$

Since $t(A)=0, t(B)=T, t_{0}$ is standard, and $t^{\prime}(A)=t^{\prime}(B),(23)$ follows easily.

(2) Now, let us consider the case $r_{A}<r_{B}$. If we suppose $x^{\prime}(t)$ has a jump at $t_{0} \gg 0$, the inverse function $t(x)$ has only a boundary layer at $x=A$ if $t_{0}=T$ and $t(x)$ has two boundary layers if $0 \ll t_{0} \ll T$. Then, in both cases, the jump at $x=A$ has $t_{+}=t_{0}$ as the extremity, with $t_{0} \gg t(A)=0$, and, by Theorem $3.5(2), t^{\prime}(A) \varepsilon^{1 /\left(r_{A}+q-1\right)}=@ \gg$. Since $t^{\prime}(A)=t^{\prime}(B)$ because $G(B)=0$, we have $t^{\prime}(A) \varepsilon^{1 /\left(r_{A}+q-1\right)}=t^{\prime}(B) \varepsilon^{1 /\left(r_{A}+q-1\right)}$.

But $t^{\prime}(B) \varepsilon^{1 /\left(r_{B}+q-1\right)}=£$ where $£$ is either an appreciable value if $0 \ll t_{0} \ll T$ (that is, if $t(x)$ has also a boundary layer at $x=B$ ) or an infinitesimal value if $t_{0}=T$ (if $t(x)$ has only a boundary layer at $x=A$ ). Then $t^{\prime}(A) \varepsilon^{1 /\left(r_{A}+q-1\right)}=£ \varepsilon^{\left(r_{B}-r_{A}\right) / d}$, where $d=\left(r_{A}-q+1\right)\left(r_{B}+q-1\right)$, and finally, as $r_{A}<r_{B}$, we have $t^{\prime}(A) \varepsilon^{1 /\left(r_{A}+q-1\right)} \simeq 0$, 
which is absurd. In the same way we prove that $x(t)$ has a boundary layer at $t_{0}=T$ if $r_{A}>r_{B}$. The stated estimate of $x^{\prime}(B)$ follows from (25) or (26).

Remark 4.1. In the theorem we also treat the turning points $A$ and $B$ as the minimum of $G(x)$ if they are inflection points. Theorem 4.1 states that if the turning points are of the same order of tangency, problem $P_{q}$ has a solution $x(t)$ with a shock at $t_{0}$ which joins the two states of equilibrium $e_{1}=A$ and $e_{2}=B$ :

$$
x(t) \simeq \begin{cases}A, & 0 \leq t \ll t_{0}, \\ B, & t_{0} \ll t \leq T .\end{cases}
$$

The value of $t_{0}$ given by (23) is the standard part of the shock location, that is, the first term in the asymptotic expansion of the shock location. The position $t_{0}$ of the shock, which depends only on the order $r$ of both turning points and on the values of $\alpha_{e}=1 /\left|g^{(r)}(e)\right|^{1 /(r+1)}$ for $e=A, B$, is the same for all values of $1 \leq q \leq 2$ and also for $q=0$. Only the thickness of the shock at $t_{0}$, which (see [6]) is defined by $\xi=\{t: A \ll x(t) \ll B\}$, depends on the values of $q$. In this case, $\xi_{q}=\left\{t: t-t_{0}=£ \varepsilon^{1 /(2-q)}, £\right.$ limited $\}$ if $q<2$, and $\xi_{q}=\left\{t:\left|t-t_{0}\right|<e^{-1 / £ \varepsilon}, £>0\right\}$ if $q=2$. These results extend those of O'Malley [15] for the quasilinear problem, $q=1$, and those of O'Malley [14] and Lutz and Goze [10] for the case $q=0$.

In the case of turning points of different orders of tangency, the higher order point dominates in the behavior of the solution and we have only a boundary layer at $t_{0}=0$ if $r_{A}<r_{B}$ (or at $t_{0}=T$ if $r_{A}>r_{B}$ ).

Remark 4.2. When we consider the boundary value problem $P_{q}$ in some standard interval $[a, b]$ and the same assumptions as in Theorem 4.1 are made, the estimate of the shock location $t_{0}$ is given by

$$
t_{0}=\frac{b+a \alpha_{B} / \alpha_{A}}{1+\alpha_{B} / \alpha_{A}}
$$

4.2. Boundary and internal behaviors. If the minimum value of $G(x)$ in $[A, B]$ is attained at $e_{1}$ and $e_{2}$ where at least one of them is an interior value of the interval $[A, B]$, the trajectory which joins $A$ with $B$ in the observability plane will look like Figure 4.1, where $V \simeq G(x)-G\left(e_{1}\right)$.

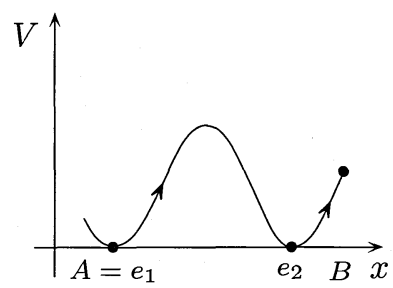

(i)

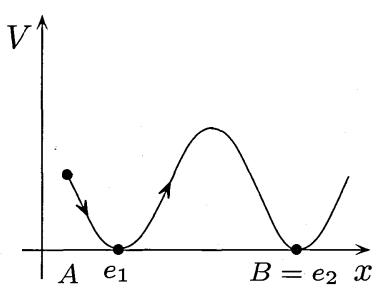

(ii)

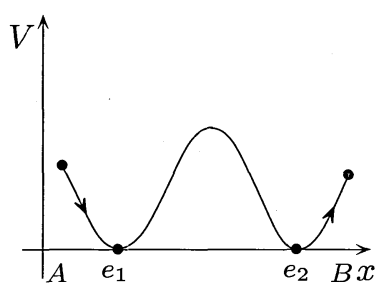

(iii)

FIGURE 4.1 

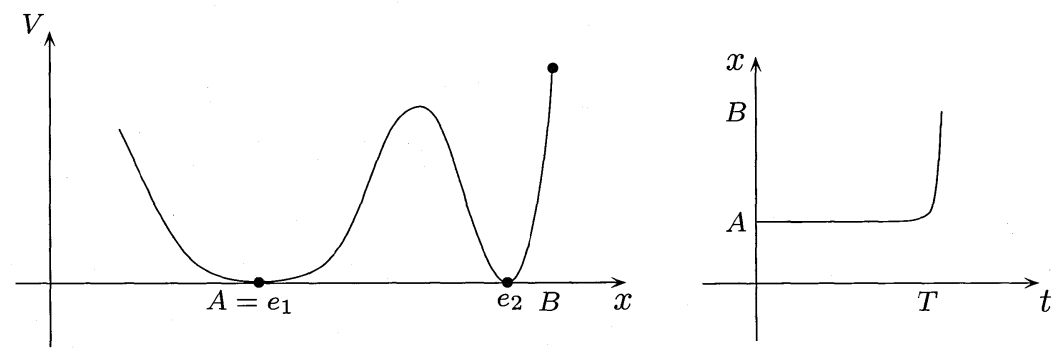

Figure 4.2

In this case, for turning points of different orders, we once again have that all the time will be spent at the turning point of higher order and we will have only boundary layer behavior. The solution will involve two boundary layers only when the higher order turning point is an interior value of $[A, B]$. If the higher order transition point is the endpoint $A$ (see Figure 4.2), then the limiting solution will be $x(t) \simeq A$ except for a terminal layer. The opposite situation (with respect to the time $t$ ) will occur if $B$ is the zero of higher order; that is, we will obtain the limiting solution $x(t) \simeq B$ except near $t=0$.

For equal order turning points, the solution has an internal layer at $t_{0}$ which joins $e_{1}$ with $e_{2}$. In order to satisfy the boundary conditions, the solution also involves one boundary layer at $t=T$ for case (i) of Figure 4.1, at $t=0$ for case (ii) of Figure 4.1, and two boundary layers for case (iii). When at least one of the turning points is an interior value of $[A, B]$, the position of the internal layer $t_{0}$ changes. This is due to the fact that for $e_{i} \in(A, B)$ the corresponding inverse function $t(x)$ of the solution $x(t)$ exhibits a singular jump at $e_{i}$ which has both an origin $t_{-}$and an extremity $t_{+}$. The extremities of the jump at $e_{i}$ are given by Corollary 3.7, and, in this case, $t_{0}$ is given by Figure 4.3. Finally, when both $e_{1}$ and $e_{2}$ are in $(A, B)$, the position of $t_{0}$ is
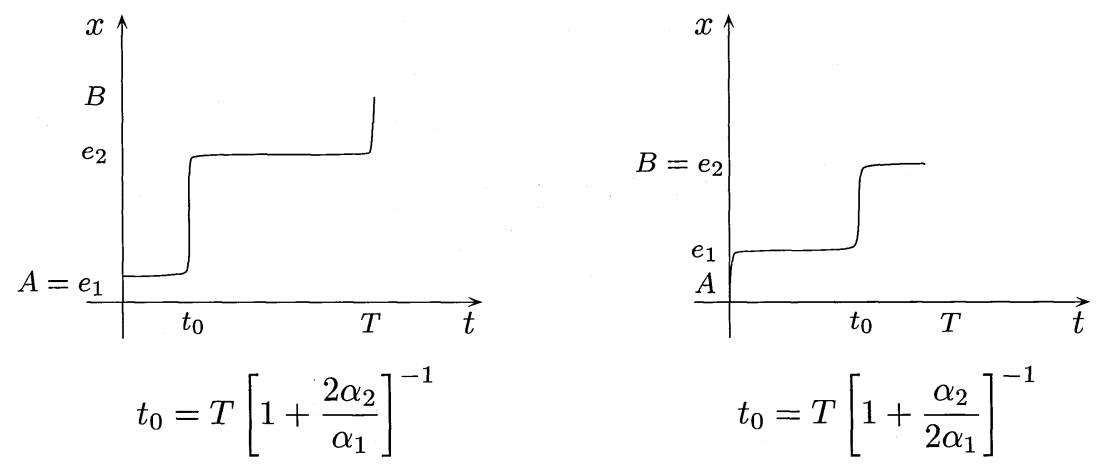

Figure 4.3

the same as when $e_{1}=A$ and $e_{2}=B$, and is given by Theorem 4.1.

In the general case of $n$ turning points of same order, $n>2$, there are $(n-1)$ internal layers and their locations are given by Figure 4.4 . 

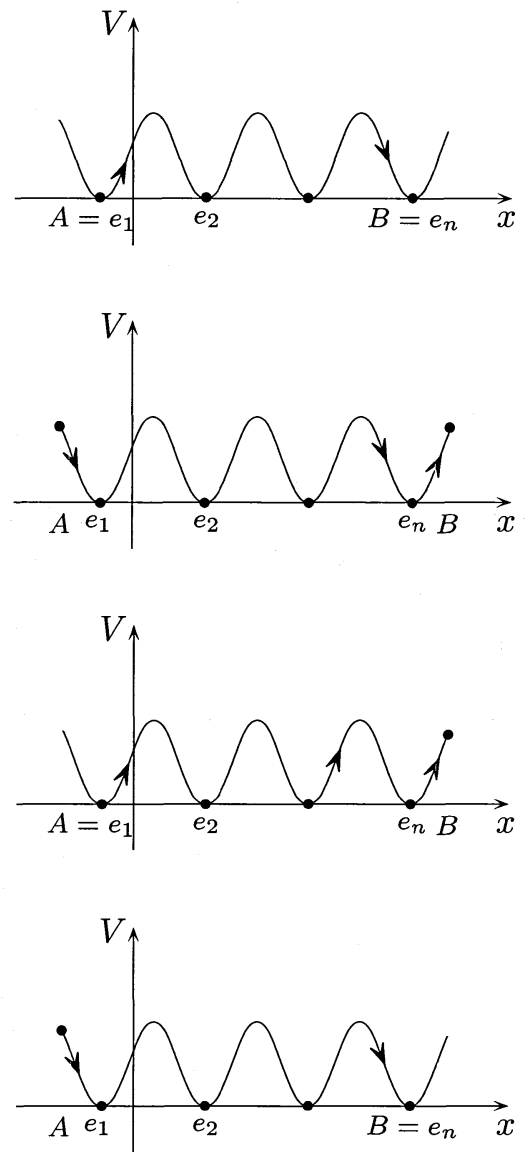

$$
\begin{aligned}
& t_{1}=T\left[1+2 \sum_{i=2}^{n-1} \frac{\alpha_{i}}{\alpha_{1}}+\frac{\alpha_{n}}{\alpha_{1}}\right]^{-1}, \\
& t_{i}=t_{i-1}+2 t_{1} \frac{\alpha_{i}}{\alpha_{1}}, \quad i=2, \ldots n
\end{aligned}
$$

(i)

$$
\begin{gathered}
t_{1}=T\left[\sum_{i=1}^{n} \frac{\alpha_{i}}{\alpha_{1}}\right]^{-1}, \\
t_{i}=t_{i-1}+t_{1} \frac{\alpha_{i}}{\alpha_{1}}, \quad i=2, \ldots n
\end{gathered}
$$

(ii)

$$
\begin{gathered}
t_{1}=T\left[1+2 \sum_{i=2}^{n} \frac{\alpha_{i}}{\alpha_{1}}\right]^{-1}, \\
t_{i}=t_{i-1}+2 t_{1} \frac{\alpha_{i}}{\alpha_{1}} \quad i=2, \ldots n
\end{gathered}
$$

(iii)

$$
\begin{gathered}
t_{1}=T\left[\sum_{i=1}^{n-1} \frac{\alpha_{i}}{\alpha_{1}}+\frac{\alpha_{n}}{2 \alpha_{1}}\right]^{-1}, \\
t_{i}=t_{i-1}+t_{1} \frac{\alpha_{i}}{\alpha_{1}}, \quad i=2, \ldots n
\end{gathered}
$$

(iv)

Figure 4.4

\section{Examples}

EXAMPLE 1.

$$
\begin{aligned}
\varepsilon x^{\prime \prime} & =x(x-1)(x+1) x^{\prime q}, \\
x(0) & =-1, \\
x(1) & =1 .
\end{aligned}
$$

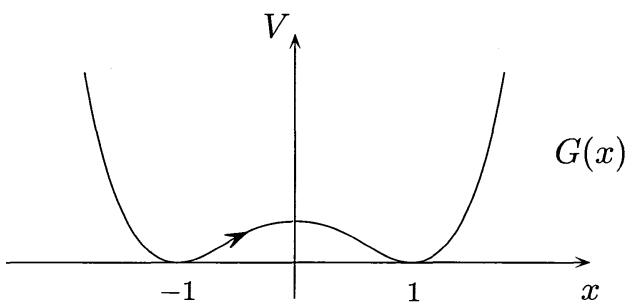

The function $G(x)=\frac{1}{4}\left(x^{2}-1\right)^{2}$ has two equal minimum values at $A=-1$ and $B=1$ of order $r=1$. We note that $\alpha_{A}=\alpha_{B}(=1 / \sqrt{2})$ since $g(x)$ is a symmetric function. Then, by Theorem 4.1, the solution $x(t)$ has an internal layer at $t_{0}=1 / 2$ 
for all the values of $1 \leq q \leq 2$. In addition, Theorem 4.1 gives the following estimate of the first derivative:

$$
x^{\prime}(0)=\left(\frac{2}{\sqrt{2}} C(1, q)\right)^{2 / q} \varepsilon^{1 / q}(1+\phi), \quad \text { for } 1 \leq q \leq 2 .
$$

Using (22) for different values of $q$, the first derivative is given explicitly by $x^{\prime}(0)=\pi^{2} \varepsilon(1+\phi)$ for $q=1, x^{\prime}(0)=(\pi / \sqrt{2})^{4 / 3} \varepsilon^{2 / 3}(1+\phi)$ for $q=3 / 2$, and $x^{\prime}(0)=\sqrt{\pi} \sqrt{\varepsilon}(1+\phi)$ for $q=2$.

We have used these values of $x^{\prime}(0)$ to compute the solution of the initial value problem at $t=0$ with a 4 -th order Runge-Kutta method. Numerical experiences show that the solution obtained provides a good approximation to the desired solution of the boundary value problem. In addition, these experiences confirm our theoretical predictions about the location of the shock layer. Table 5.1 shows the numerical values of $t_{0}$ and of $x(1)$ for some $\varepsilon$.

\begin{tabular}{|c|c|c|c|c|}
\hline \multirow{2}{*}{$q$} & \multicolumn{2}{|c|}{$\varepsilon=0.01$} & \multicolumn{2}{c|}{$\varepsilon=0.003$} \\
\cline { 2 - 5 } & $t_{0}$ & $x(1)$ & $t_{0}$ & $x(1)$ \\
\hline 1 & 0.5309 & 0.9938 & 0.5129 & 0.9992 \\
\hline $3 / 2$ & 0.5339 & 0.9906 & 0.5135 & 0.9989 \\
\hline 2 & 0.5328 & 0.9889 & 0.5169 & 0.9969 \\
\hline
\end{tabular}

Table 5.1

We exclude the case $q=0$ since the turning points are of order $r=1$ which makes $r+q-1=0$, however we can anticipate ([3], [9], [10], [14], and [17]) that the solution also has an internal layer at $t_{0}=1 / 2$ for $q=0$. In this case, the first derivative of the solution is exponentially small (see [3] or [9]).

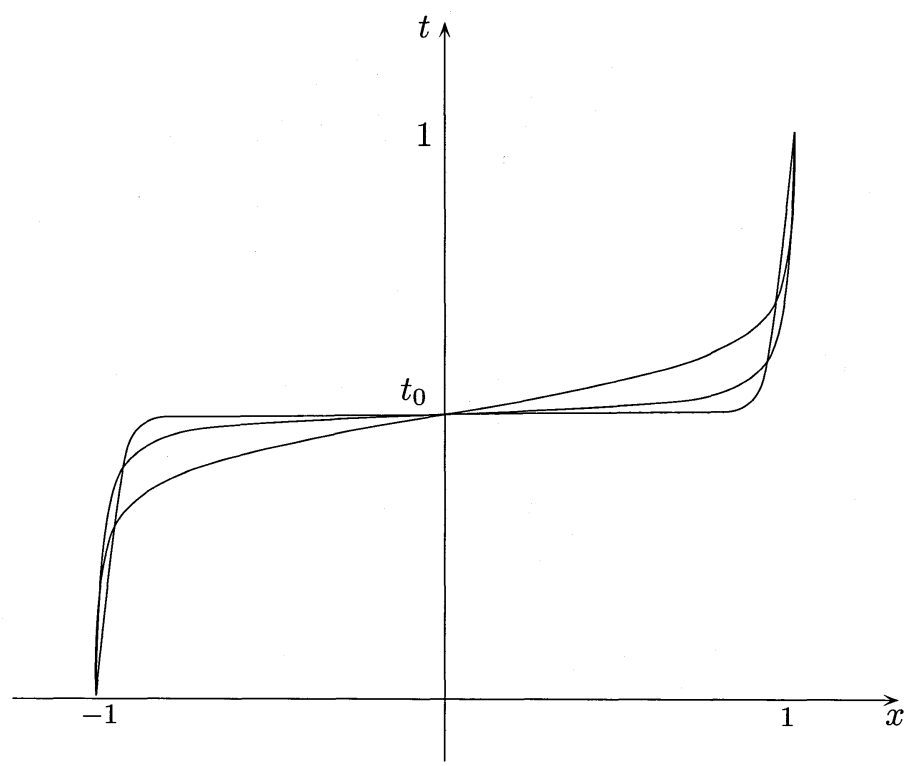

Figure 5.1 
EXAMPLE 2.

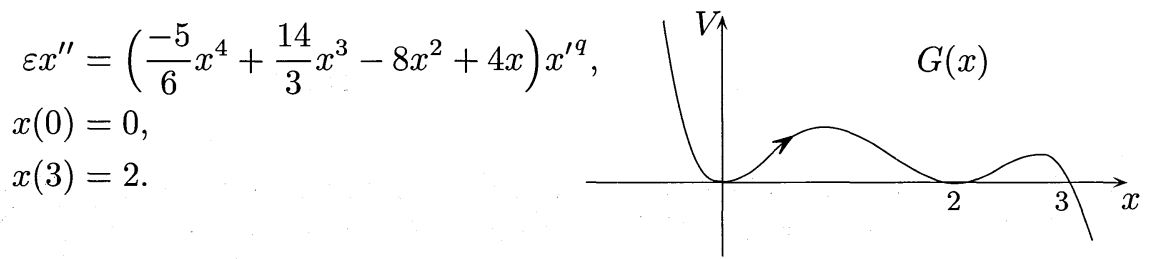

This example concerns the case of a non symmetric function $g(x)$ where $A=0$ and $B=2$ are consecutive turning points of the same order $r=1$. As $\alpha_{A}=1 / 2$ and $\alpha_{B}=\sqrt{3} / 2$, it follows from Theorem 4.1 that the solution $x(t)$ exhibits an internal layer at $t_{0}=1.09807$ for all values of $1 \leq q \leq 2$.

In this case, the first derivative is given by:

$$
x^{\prime}(0)= \begin{cases}\left(\frac{\pi}{2 \sqrt{2} t_{0}}\right)^{2} \varepsilon(1+\phi) & \text { if } q=1, \\ \left(\frac{\pi}{4 t_{0}}\right)^{4 / 3} \varepsilon^{2 / 3}(1+\phi) & \text { if } q=3 / 2, \\ \frac{\sqrt{\pi}}{2 \sqrt{2} t_{0}} \sqrt{\varepsilon}(1+\phi) & \text { if } q=2 .\end{cases}
$$

The values of $t_{0}$ and $x(3)$ obtained by using the above estimates of $x^{\prime}(0)$ in the same way as in Example 1 are shown in Table 5.2.

\begin{tabular}{|c|c|c|}
\hline \multirow{2}{*}{$q$} & \multicolumn{2}{|c|}{$\varepsilon=0.005$} \\
\cline { 2 - 3 } & $t_{0}$ & $x(3)$ \\
\hline 1 & 1.1174 & 1.999907 \\
\hline $3 / 2$ & 1.1246 & 1.999164 \\
\hline 2 & 1.1432 & 1.997799 \\
\hline
\end{tabular}

Table 5.2

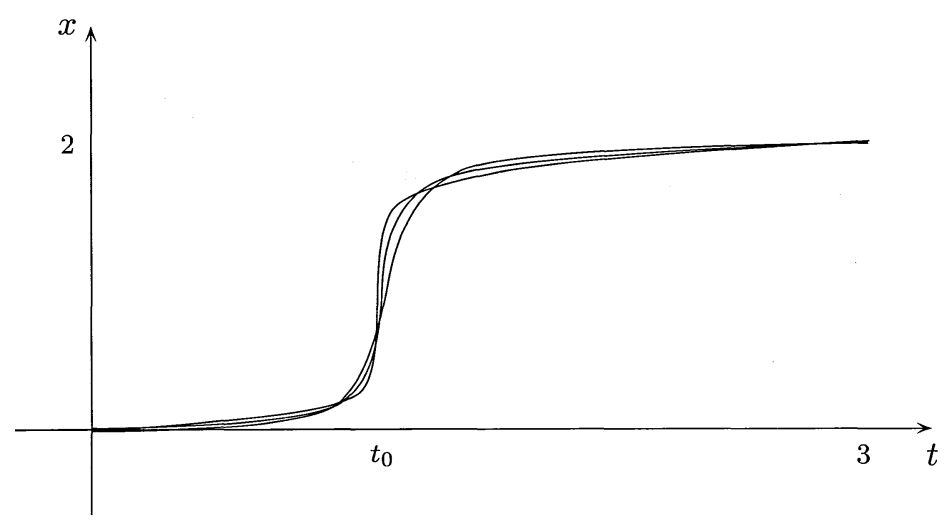

FiguRE 5.2 
EXAMPLE 3 .

$$
\begin{aligned}
\varepsilon x^{\prime \prime} & =\sin x x^{\prime q}, \\
x(0) & =A \\
x(1) & =B .
\end{aligned}
$$

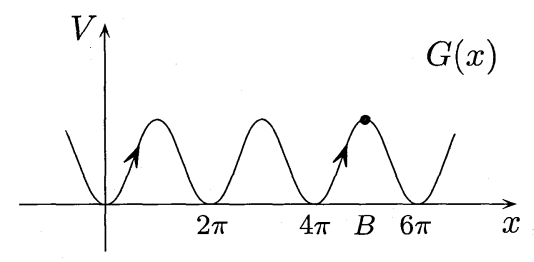

The function $G(x)=1-\cos x$ has equal minimum values at $e_{k}=2 k \pi, k \in \mathbb{N}$, each of order $r=1$.

For $A=0$ and $B=2 \pi$, the solution has an internal layer at $t_{0}=1 / 2$ for all values of $q$.

For $A=0$ and $4 \pi<B<6 \pi$, the solution has two internal layers at $t_{1}=1 / 5$ and $t_{2}=3 / 5$ and a boundary layer at $t=1$. In this case, the solution may be computed with $x^{\prime}(0)=2\left(\pi / t_{1}\right)^{2} \varepsilon$ for $q=1$ and $x^{\prime}(0)=\left(\sqrt{\pi} / \sqrt{2} t_{1}\right) \sqrt{\varepsilon}$ for $q=2$.

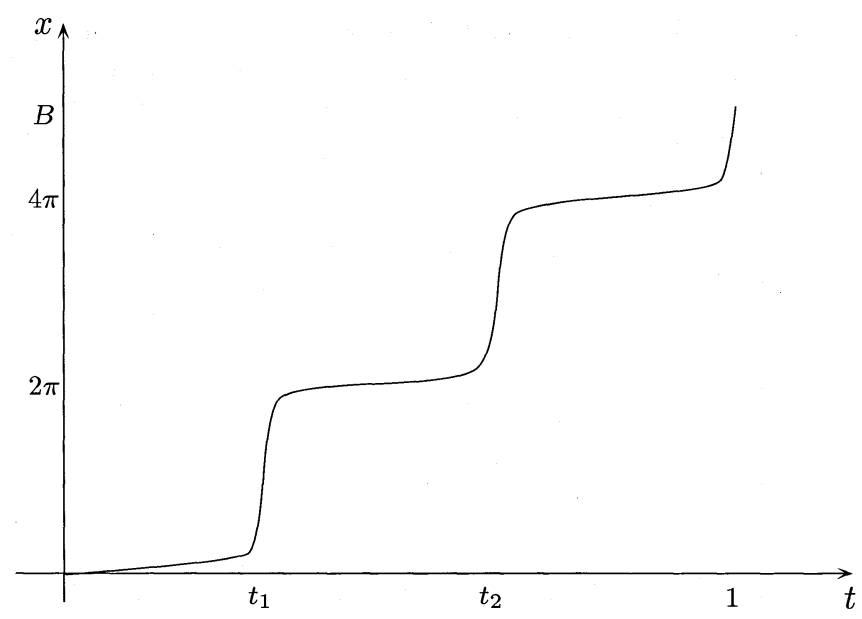

FIGURE 5.3

EXAMPLE 4.

$$
\begin{aligned}
\varepsilon x^{\prime \prime} & =\frac{d}{d x}\left(x^{2}(1-\cos x)\right) x^{\prime q}, \\
x(0) & =A \\
x(1) & =B .
\end{aligned}
$$

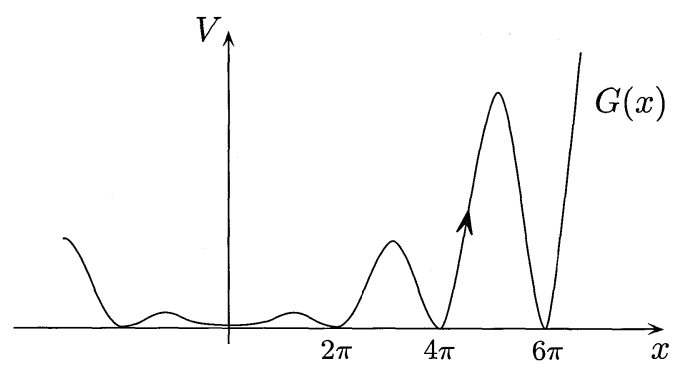

The function $G(x)=x^{2}(1-\cos x)$ has equal minimum values at $e_{k}=2 k \pi$, but $e_{0}=0$ is a zero of order $r_{0}=3$ while the others are of order $r_{k}=1, k \geq 1$. 
For $A=0$ and $B=2 k \pi, k \geq 1$, the solution has a boundary layer at $t=1$. A very interesting situation occurs when the number $k$ of turning points $e_{k}$ of order $r=1$ belonging to $[A, B]$ increases. The corresponding solution always has a boundary layer at $t=1$, but this boundary layer exhibits many deformations, as many as the value of $k$. These deformations $\mu_{k}$ are the contributions of each turning point $e_{k}$ to the trajectory when it passes through them.

This is critical from the numerical point of view. The deformations $\mu_{k}$ must be taken into account to get a good estimation of $x^{\prime}(0)$ in order to compute the solution. The values of $\mu_{k}$ which may be estimated from the relationships (20) and (21) are given by

$$
\mu_{k}=2 \alpha_{k}\left(t^{\prime}\left(e_{k}\right) \varepsilon^{1 / q}\right)^{q / 2} C(1, q)
$$

where $\alpha_{k}=1 / e_{k}$. Since $e_{0}$ is a turning point of order $r_{0}=3$, Theorem 4.1 shows that $t^{\prime}\left(e_{0}\right)=@ / \varepsilon^{1 /(2+q)}$. Since $t^{\prime}\left(e_{0}\right)=t^{\prime}\left(e_{k}\right)$, it follows that the deformations are of order $\mu_{k}=O\left(\varepsilon^{1 /(2+q)}\right)$.

For example, if $q=1, A=0$, and $B=6 \pi$, the deformations are given by $\mu_{k}=(@ / k) \varepsilon^{1 / 3}, k=1,2,3$ (with $\mu_{3}$ halved as $B=6 \pi$ ). Then a good estimate of $x^{\prime}(0)$ must be obtained from (24) with $T=1-\left(\mu_{1}+\mu_{2}+\mu_{3} / 2\right)$.

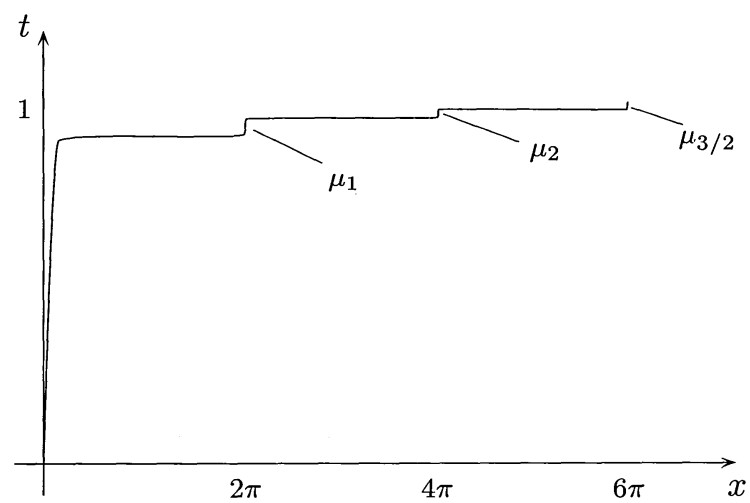

FiguRE 5.4

\section{EXAMPLE 5.}

$$
\begin{aligned}
\varepsilon x^{\prime \prime} & =-x(x-1)^{2}(x+1)^{2} x^{\prime q}, \\
x(0) & =-1, \\
x(1) & =1 .
\end{aligned}
$$

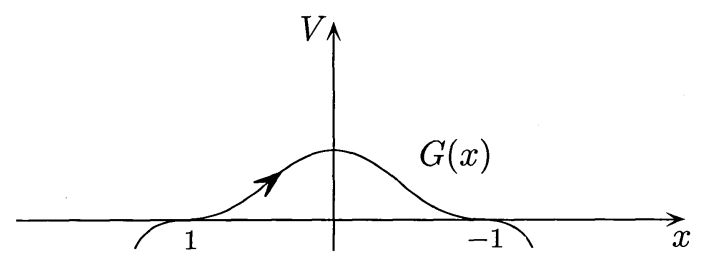

The function $G(x)=x^{2}\left(-3+3 x^{2}-x^{4}\right) / 6+1 / 6$ has two inflection points at $A=-1$ and $B=1$ and $\alpha_{A}=\alpha_{B}=1 / 2$. We consider these values of $A$ and $B$ as the two equal minimum values of $G(x)$ of order $r=2$. Then it follows from Theorem 4.1 that 
the solution $x(t)$ exhibits an internal layer at $t_{0}=1 / 2$ for all values of $q$. The solution may be computed by considering

$$
x^{\prime}(0)= \begin{cases}\frac{\sqrt{3}}{3 \sqrt{8}}\left(\frac{\Gamma^{3}(1 / 3)}{\pi t_{0}}\right)^{3} \varepsilon, & \text { if } q=0, \\ \frac{\sqrt{2}}{3^{7 / 4}}\left(\frac{\pi}{t_{0}}\right)^{3 / 2} \sqrt{\varepsilon}, & \text { if } q=1, \\ 36^{-1 / 3} \frac{\Gamma(1 / 3)}{t_{0}} \varepsilon^{1 / 3}, & \text { if } q=2 .\end{cases}
$$

\section{References}

1. A. Bohé, Free layers in a singularly perturbed boundary value problem, SIAM J. Math. Anal. 21 (1990), 1264-1280.

2. ordinaires, Thèse de Doctorat, Université de Paris 7, Paris, France, 1991.

3. _ Free layers and singular jumps in some superquadratic equations, in preparation.

4. V. F. Butuzov and A. B. Vasil'eva, The asymptotic theory of contrasting spatial structures, USSR Comp. Math. and Math. Physics 28 (1988), 26-36.

5. F. Diener, Méthode du plan d'observabilité, Thèse, Université Louis Pasteur, Strasbourg, France, 1981.

6. $533-559$.

7. F. Diener and G. Reeb, Analyse Non Standard, Hermann, Paris, 1989.

8. P. C. Fife, Dynamics of Internal Layers and Diffusive Interfaces, SIAM, Philadelphia, 1988.

9. W. L. Kath, C. Knessl, and B. J. Matkowsky, A Variational approach to nonlinear singularly perturbed boundary value problems, Stud. Appl. Math. 77 (1987), 61-88.

10. R. Lutz and M. Goze, Nonstandard Analysis, Lectures Notes in Math. Vol. 881, Springer-Verlag, New York, 1981.

11. B. J. Matkowsky, Singular perturbations, stochastic differential equations, and applications, in Singular Perturbations and Asymptotics, R. E. Meyer and S. V. Parter, eds., Academic Press, New York, 1980, 109-147.

12. D. W. McLaughlin, Path integrals, asymptotics and singular perturbations, J. Math. Phys. 13 (1972), 786-796.

13. _ Complex time, contour independent path integrals, and barrier penetration, J. Math. Phys. 13 (1972), 1099-1108.

14. R. E. O'Malley, Jr., Phase-plane solutions to some singular perturbation problems, J. Math. Anal. Appl. 54 (1976), 449-466.

15. _ Singular perturbations, asymptotic evaluation of integrals, and computational challenges, in Asymptotic Analysis and the Numerical Solution of Partial Differential Equations, Hans G. Kaper and Marc Garbey, eds., Marcel Dekker, New York, 1991, 3-16.

16. I. Van Den Berg, Nonstandard Asymptotics Analysis, Lecture Notes in Math. Vol. 1249, SpringerVerlag, New York, 1987.

17. M. J. Ward, Eliminating indeterminacy in singularly perturbed boundary value problems with translation invariant potentials, Stud. Appl. Math. 87 (1992), 95-134.

U. F. R. De Matheḿatiques, Université de Paris 7, 75251 Paris Cedex 05, France 